\title{
CHERN NUMBERS AND ORIENTED HOMOTOPY TYPE
}

\author{
BY PETER J. KAHN ${ }^{1}$
}

Communicated by P. E. Conner, Jr, May 25, 1967

1. Statement of results. This note announces the solution of the following problem, a more precise version of which appears in \$2:

(1) Determine the linear combinations of rational Chern numbers (of almost-complex manifolds) that are invariants of oriented homotopy type (of almost-complex manifolds).

Milnor, who posed the problem, conjectured that every such homotopy invariant could be expressed as a rational linear combination of the Euler Characteristic and the Index.

THEOREM 1. Milnor's conjecture is true.

We obtain variations on problem (1) by some simple alterations:

(2) Replace the word "homotopy" in (1) by "diffeomorphism."

TheOREM 2. Modulo those linear combinations of rational Chern numbers that can be expressed in terms of Pontrjagin numbers, the only combinations that are invariants of oriented diffeomorphism type are the rational multiples of the Euler Characteristic.

(3) Stabilize (1). That is, consider the same problem with reference to the class of stably almost-complex manifolds. According to Milnor (see [2, pp. 122-127]), this does not affect the possible configurations of Chern numbers. However, it does strictly decrease the number of independent combinations that are oriented homotopy invariant. Stong has conjectured that multiples of the Index are the only oriented homotopy invariant combinations.

THEOREM $1_{s}$. Stong's conjecture is true.

(4) Finally, we may stabilize (2).

THEOREM 2 . Every linear combination of rational Chern numbers (of stably almost-complex manifolds) that is an invariant of oriented diffeomorphism type (of stably almost-complex manifolds) can be expressed as a linear combination of rational Pontrjagin numbers.

We may restate Theorem $2_{s}$ in the following way: Let $\Omega_{*}^{U}$ and $\Omega_{*}^{S O}$ denote the complex and oriented cobordism rings, respectively, and let

${ }^{1}$ This research was partly supported by NSF grant GP 6556. 


$$
\Omega_{*}^{U} \otimes Q \stackrel{\pi}{\rightarrow} \Omega_{*}^{\text {so }} \otimes Q
$$

be the canonical surjection. Let $D_{i}^{S}$ denote the set of all classes in $\Omega_{i}^{U}$ of the form $\left[M_{1}^{i}\right]-\left[M_{2}^{i}\right]$, where $M_{1}^{i}, M_{2}^{i}$ are stably almost-complex manifolds that are oriented diffeomorphic. It is easy to show that $D_{i}^{S}$ is a subgroup of $\Omega_{t}^{U}$ and that $D_{*}^{S}$ is an ideal in $\Omega_{*}^{U}$. Clearly, $D_{*}^{S} \otimes Q$ $C \operatorname{ker} \pi$. Theorem $2_{S}$ is equivalent to the assertion that

$$
D_{*}^{S} \otimes Q=\operatorname{ker} \pi \text {. }
$$

REMARK. Since Pontrjagin numbers are invariants of oriented topological type (of smooth, oriented manifolds)-this result is due to Novikov [5]-Theorem 2 and Theorem $2_{S}$ still hold when the word "diffeomorphism" is replaced by "homeomorphism."

2. Reformulation of the problems. The graded ring $H^{*}(B U ; Q)$ is a polynomial ring on the universal Chern classes $c_{k} \in H^{2 k}(B U ; Q)$. We shall identify it in the usual way $([2],[7])$ with the graded vectorspace dual of $\Omega_{*}^{U} \otimes Q$ : monomials in the $c_{k}$, then, become universal Chern numbers qua linear functionals.

Corresponding to each problem (1), (2), (3), (4), we define a certain ideal $\mathfrak{F}_{*}, \mathfrak{D}_{*}, \mathfrak{F}_{*}^{S}, \mathfrak{D}_{*}^{S}$, respectively, contained in $\Omega_{*}^{U}$. We have already defined $D_{*}^{S}$ in $\$ 1$; for brevity, we define only $\mathcal{H}_{*}$ here, the other two ideals being defined analogously. Let $\mathfrak{H C}_{2 k} \subset \Omega_{2 k}^{U}$ consist of all complex cobordism classes of the form $\left[M_{1}^{2 k}\right]-\left[M_{2}^{2 k}\right]$, where $M_{1}^{2 k}, M_{2}^{2 k}$ are almost-complex manifolds of the same oriented homotopy type. That $\mathcal{H}_{*}$ is an ideal in $\Omega_{*}^{U}$ is easily verified.

The annihilator of the subspace $\mathcal{H C}_{2 k} \otimes Q \subset \Omega_{2 k}^{U} \otimes Q$ is precisely the set of rational linear combinations of (complex) $k$-dimensional Chern numbers (of almost-complex manifolds) that are invariants of oriented homotopy type. Problem (1) then may be reformulated as:

$\left(1^{\prime}\right)$ Determine a basis of the annihilator of $\mathfrak{F}_{2 k} \otimes Q, k=1,2,3, \cdots$.

The Euler Characteristic and Index of $2 k$-dimensional almostcomplex manifolds - the latter is defined only for even $k$-are well known, linearly independent members of this annihilator. Milnor's conjecture is that they form a basis of the annihilator. Therefore, Theorem 1 is equivalent to the assertion:

TheOREM $1^{\prime}$. Co-dimension $\mathfrak{H}_{2 k} \otimes Q \leqq 1, \quad k \quad$ odd,

$$
\leqq 2, k \text { even. }
$$

To prove this theorem, we make use of the ring structure of $\Omega_{*}^{U} \otimes Q$ (determined by Milnor [2]): it is generated as a polynomial ring by classes $y_{k} \in \Omega_{2 k}^{U} \otimes Q, k=1,2,3, \cdots$. Any sequence $\left\{y_{k}\right\}$ with $y_{k}$ 
$\in \Omega_{2 k}^{U} \otimes Q$ will be called a generating sequence, provided that the $y_{k}$ generate $\Omega_{*}^{U} \otimes Q$ as a polynomial ring. We prove:

THEOREM $1^{\prime \prime}$. There is a generating sequence $\left\{y_{k}\right\}$ with $y_{1} y_{2} \in \mathcal{F C}_{6} \otimes Q$ and $y_{k} \in \mathcal{F}_{2 k} \otimes Q, k \geqq 3$.

Using the fact that $\mathfrak{H C}_{*} \otimes Q$ is an ideal, it is easy to show that Theorem $1^{\prime \prime}$ implies Theorem $1^{\prime}$.

Similar reformulations of the other problems lead to the following theorems, which imply their counterparts in $\$ 1$.

THEOREM $2^{\prime \prime}$. There is a generating sequence $\left\{y_{k}\right\}$ with $y_{1} y_{k}$ $\in D_{2 k+2} \otimes Q$, if $k$ is even, and $y_{k} \in D_{2 k} \otimes Q$, if $k$ is odd and $k \geqq 3$.

THEOREM $1_{S}^{\prime \prime}$. There is a generating sequence $\left\{y_{k}\right\}$ with $y_{k} \in \mathcal{J}_{2 k}^{S} \otimes Q$, for $k \neq 2$.

THEOREM $2 \prime \prime s$. There is a generating sequence $\left\{y_{k}\right\}$ with $y_{k} \in D_{2 k}^{S} \otimes Q$, if $k$ is odd.

To construct the low-dimensional ring generators, we make use of results of [8]. For large even $k$, the ring generators $y_{k}$ are obtained by using certain results of W. C. Hsiang [3]. For large odd $k$, we make use of some examples pointed out to us by Stong (for the stable theorems), and we use results of Adams [1] and Sanderson [6] on $[K U]^{\sim}\left(C P^{k}\right)$ and $[K O]^{\sim}\left(C P^{k}\right)$ (for the unstable theorems). Techniques described in [4] for killing certain obstructions are also used for the unstable theorems.

\section{REFERENCES}

1. J. F. Adams, Vector fields on spheres, Ann. of Math. 75 (1962), 603-632.

2. F. Hirzebruch, Komplexe Mannigfaltigkeiten, Proc. Internat. Congr. Math. 1958, pp. 119-136, Cambridge Univ. Press, New York, 1960.

3. W. C. Hsiang, $A$ note on free differentiable actions of $S^{1}$ and $S^{3}$ on homotopy spheres, Ann. of Math. 83 (1966), 266-272.

4. P. J. Kahn, Obstructions to extending almost $X$-structures, preprint, Cornell University, Ithaca, New York.

5. S. P. Novikov, Izv. Akad. Nauk SSSR 30 (1966), 207-246. (Russian)

6. B. J. Sanderson, Immersions and embeddings of projective spaces, Proc. London Math. Soc. (3) 14 (1964), 137-153.

7. R. E. Stong, Relations among characteristic numbers. I, Topology 4 (1965), 267-281.

8. C. T. C. Wall, Classification problems in differential topology. V, Inventiones Mathematicae 1 (1966), 355-374.

CORNell University 